Take the online multiple choice questions associated with this article (see page 535)
See end of article for authors' affiliations

Correspondence to: Dr Perry Elliott, The Heart Hospital, 16-18 Westmoreland Street, London, WIG 8PH, UK; pelliott@doctors.org.uk ysosomal storage disorders (LSD) comprise a group of more than 40 diseases caused by a deficiency of lysosomal enzymes, membrane transporters or other proteins involved in lysosomal biology. The predominant inheritance pattern is autosomal recessive except for Anderson-Fabry disease, glycogen storage disease (GSD) type IIb (Danon disease) and mucopolysaccharidosis (MPS) type II (Hunter disease). While the metabolic defects affect all cells, clinical organ involvement usually occurs only in the presence of substrate excess or metabolic pathway activation. Cardiac disease is particularly important in lysosomal glycogen storage diseases (Pompe and Danon disease), mucopolysaccharidoses and in glycosphingolipidoses (Anderson-Fabry disease). Various disease manifestations may be observed including hypertrophic and dilated cardiomyopathy, coronary artery disease and valvular disease (table 1 ).

\section{ANDERSON-FABRY DISEASE}

Anderson-Fabry disease (AFD, synonyms Fabry disease, $\alpha$-galactosidase A deficiency, angiokeratoma corporis diffusum) is an X-linked LSD caused by mutations in the gene encoding the lysosomal enzyme $\alpha$-galactosidase A. The resultant deficiency in $\alpha$-galactosidase A activity leads to intralysosomal accumulation of neutral glycosphingolipids, mainly globotriaosylceramide $\left(\mathrm{Gb}_{3}\right)$, in various organ systems. The disease is characterised by progressive clinical manifestations and premature death from renal failure, stroke and cardiac disease.

\section{Epidemiology}

The incidence of AFD has been estimated at 1 in 40000 to 1 in 117000 live births for males. ${ }^{1}$ Recently, studies in high risk patient cohorts suggest that it is much more common. The reported prevalence of AFD in patients with end-stage renal disease on haemodialysis ranges between $0.2-$ $1.2 \%$; in patients with cryptogenic stroke the prevalence may be as high as $4.9 \%$ in men and $2.8 \%$ in women. The prevalence of AFD in patients with heart disease varies depending on the population studied. In a survey of male patients with unexplained left ventricular hypertrophy (LVH) attending an echocardiography clinic, 3\% had biochemical evidence for $\mathrm{AFD}^{2}$; in a second analysis of male patients attending a specialist hypertrophic cardiomyopathy clinic, $4 \%$ had diagnostic plasma levels of $\alpha$-galactosidase A. ${ }^{3}$ Even higher prevalence figures rates have been reported in studies using endomyocardial biopsy as a screening tool. ${ }^{4}$

\section{Molecular genetics}

The $\alpha$-galactosidase A gene consists of seven exons located on the long arm of the $\mathrm{X}$ chromosome (Xq22.1) that encode a $101 \mathrm{kD}$ homodimeric glycoprotein. Over 250 mutations have been described in all seven exons, the majority of which are missense point mutations. Individual mutations are often limited to one or few families ("private mutations"). ${ }^{5}$ Reduced enzyme activity occurs by several mechanisms including abnormal or unstable protein folding, perturbation to active binding sites and defective enzyme tracking to the lysosome. ${ }^{1}$

\section{Clinical presentation}

The most common clinical features in classical AFD are summarised in table 2. Although many symptoms occur in childhood, correct diagnosis is delayed by as much as 14 years in males and 16 years in females. ${ }^{1}$ In males, symptoms start usually in the first decade of life with acroparesthesiae and pain, febrile crises, hypohidrosis, heat intolerance, gastrointestinal disturbance and the development of cutaneous angiokeratomata. From the second decade onwards patients develop proteinuria and neurological manifestations, including vestibular and hearing disturbance and autonomic dysfunction. Cardiac involvement is present early in life but is not detected clinically until the third or fourth decade (fig 1 ). ${ }^{1}$ 
Table 1 Lysosomal storage disease causing cardiac disease

\begin{tabular}{|c|c|c|}
\hline Disease group and subtypes & General manifestations & Cardiac manifestations \\
\hline \multicolumn{3}{|l|}{ Glycogen storage diseases (lysosomal) } \\
\hline & Autosomal recessive & Massive LVH and RVH, cardiac failure (only in the infantile form) \\
\hline & $\begin{array}{l}\text { Myopathy, hypotonia, hepatomegaly, } \\
\text { macroglossia cardiopulmonary failure, }\end{array}$ & Short PR, broad QRS; endomyocardial fibrosis \\
\hline \multirow[t]{2}{*}{ Type Ilb (Danon disease, LAMP-2 deficiency) } & $X$-linked & Hypertrophic cardiomyopathy, isolated cardiac variants, short \\
\hline & Myopathy, mental retardation & PR, progressive conduction system disease \\
\hline \multicolumn{3}{|l|}{ Mucopolysaccharidoses } \\
\hline \multirow{2}{*}{$\begin{array}{l}\text { IH (Hurler) } \\
\text { IS (Scheie) }\end{array}$} & Autosomal recessive & Valvular involvement (thickening, regurgitation, stenosis); \\
\hline & X-linked - MPS II (Hunter) & endomyocardial infiltration; interstitial infiltration-fibrosis; \\
\hline & Dysmorphic features, organomegaly, decreased & hypertrophy; systolic dysfunction - dilated cardiomyopathy (less \\
\hline III (Sanfilippo) & joint mobility, bone deformities, loss of motor & frequent); coronary artery infiltration-stenosis; aortic stenosis \\
\hline $\begin{array}{l}\text { IV (Morquio) } \\
\text { VI (Maroteaux-Lamy) }\end{array}$ & skills, mental retardation, corneal clouding, & (abdominal); arterial hypertension \\
\hline \multicolumn{3}{|c|}{ recurrent otitis or pneumonia, hearing loss } \\
\hline \multicolumn{3}{|l|}{ IX (Natowicz) } \\
\hline \multicolumn{3}{|l|}{ Sphingolipidoses } \\
\hline \multirow{2}{*}{$\begin{array}{l}\text { Gaucher disease ( } \beta \text {-glucocerebrodiase) } \\
\text { Chronic non-neuronopathic (type I) } \\
\text { Acute (type II) }\end{array}$} & Autosomal recessive & Pulmonary hypertension, cor pulmonale; \\
\hline & $\begin{array}{l}\text { Gaucher cells - lipid laden macrophages } \\
\text { Hepatosplenomegaly, anaemia, }\end{array}$ & pericardial effusion (rare); valvular involvement (rare) \\
\hline \multirow{5}{*}{$\begin{array}{l}\text { Chronic neuronopathic (type III) } \\
\text { Niemann Pick disease (acid sphingomyelinase) } \\
\text { Type A } \\
\text { Type B }\end{array}$} & Neurodegeneration (neuronopathic forms) & \\
\hline & Autosomal recessive & Endomyocardial fibrosis (very rare) \\
\hline & Early onset, neurological involvement, & \\
\hline & hypotonia, psychomotor retardation (type A), & \\
\hline & $\begin{array}{l}\text { hepatosplenomegaly, pancyłopenia, } \\
\text { pulmonary involvement }\end{array}$ & \\
\hline \multirow[t]{3}{*}{ Anderson-Fabry disease ( $\alpha$-galactosidase A) } & $X$-linked & Cardiac hypertrophy; short PR, progressive conduction system \\
\hline & Multiorgan involvement & dysfunction, arrhythmias; valvular involvement; coronary \\
\hline & & involvement (decreased coronary reserve) \\
\hline
\end{tabular}

\section{Disease in females}

Despite early autopsy reports demonstrating clinically significant disease in female heterozygotes, for a long period of time women were considered only as disease carriers. However, many studies have shown that affected women experience symptoms similar to hemizygous males, albeit in a milder form with a delayed onset and slower progression compared to men; similarly, the frequency of end-stage renal disease is much lower and the median cumulative survival greater ( 70 years vs

Table 2 Main extracardiac clinical manifestations of Anderson Fabry disease

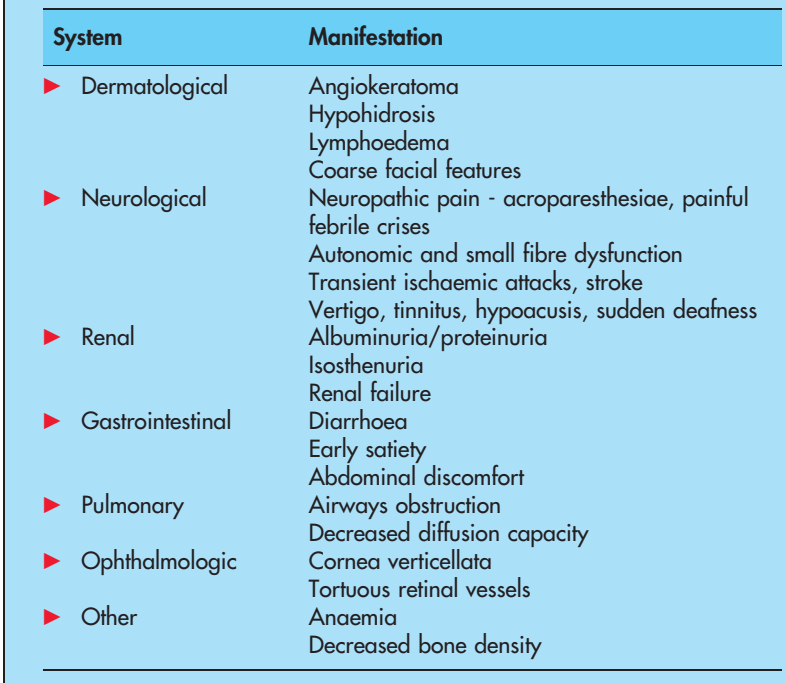

50 years) in women. ${ }^{1}$ Disease expression in female patients is attributed to random $\mathrm{X}$ chromosome inactivation and the incapacity of cells expressing the wild-type allele to crosscorrect the metabolic defect. $^{5}$

\section{Pathogenesis of cardiac disease in AFD}

Cross-sectional data in patients of different ages suggest that disease evolution in the heart is characterised initially by myocardial hypertrophy; as the disease progresses interstitial abnormalities and replacement myocardial fibrosis become important. This correlates well with observations of relatively mild diastolic dysfunction in early stages of the disease progressing to systolic and severe diastolic ventricular impairment in advanced disease. ${ }^{6}$

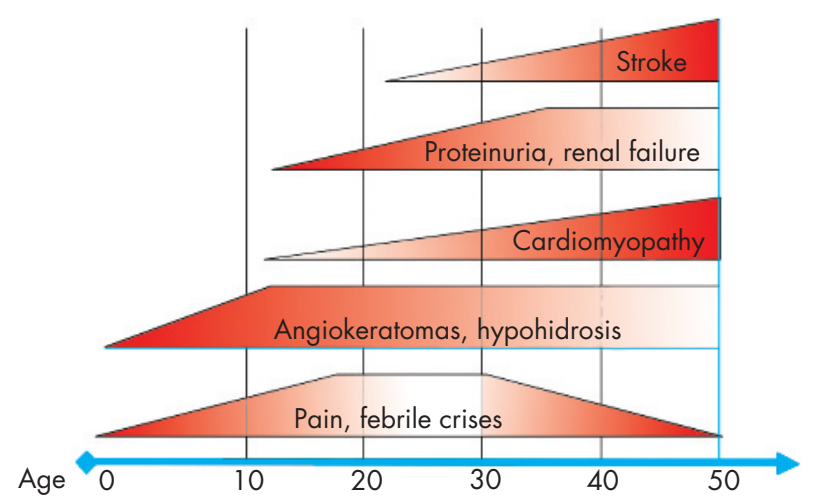

Figure 1 Typical disease course in a classically affected male hemizygote with Anderson-Fabry disease. 
Cardiac disease in AFD is associated with $\mathrm{Gb}_{3}$ accumulation in all cellular components of the heart, including cardiomyocytes, conduction system cells, valvular fibroblasts, endothelial cells and vascular smooth muscle cells. In women, a mosaic pattern caused by random X chromosome inactivation can be observed. ${ }^{5}$ Myocytes are vacuolated and hypertrophied, but

unlike familial hypertrophic cardiomyopathy, myofibrillar disarray is not prominent. Lysosomal inclusions are present within myofibrils and vascular structures (figs 2 and 3); fibrosis is evident within the mid-myocardial layers and the posterolateral segments of the left ventricle.

While $\mathrm{Gb}_{3}$ accumulation is the most prominent feature histologically, it represents only $1-2 \%$ of the total cardiac mass. ${ }^{6}$ Therefore, it is likely that disease in the heart results from activation of other signalling pathways that lead to hypertrophy, apoptosis, necrosis and fibrosis.

Circumstantial evidence suggests that myocardial ischaemia in the absence of significant disease of epicardial arteries may contribute to disease progression. The underlying mechanisms probably include the increased oxygen demand of the hypertrophied muscle, decreased capillary density and increased diastolic filling pressures; $\mathrm{Gb}_{3}$ deposition in endothelial and smooth muscular cells of small arterioles and capillaries is another factor. ${ }^{8}$

\section{Specific cardiac abnormalities in AFD \\ The "cardiac variant"}

A number of reports have suggested that some patients with residual $\alpha$-galactosidase A activity (approximately $1-5 \%$ of normal values) present in middle age with LVH and conduction disease, in the absence of other classical disease manifestations. Some patients with this "cardiac variant" had proteinuria, but did not develop end-stage renal disease; they also differed from classical AFD patients in that they did not have glycosphingolipid deposits in vascular endothelium. ${ }^{23}$ Contemporary studies suggest that if it exists at all, the cardiac variant of AFD is extremely rare, as rigorous clinical characterisation reveals organ involvement elsewhere. Nevertheless, clinical presentation in patients with residual activity can be dominated by cardiovascular involvement, meaning that AFD should be considered in the differential diagnosis of otherwise unexplained ventricular hypertrophy (table 3).
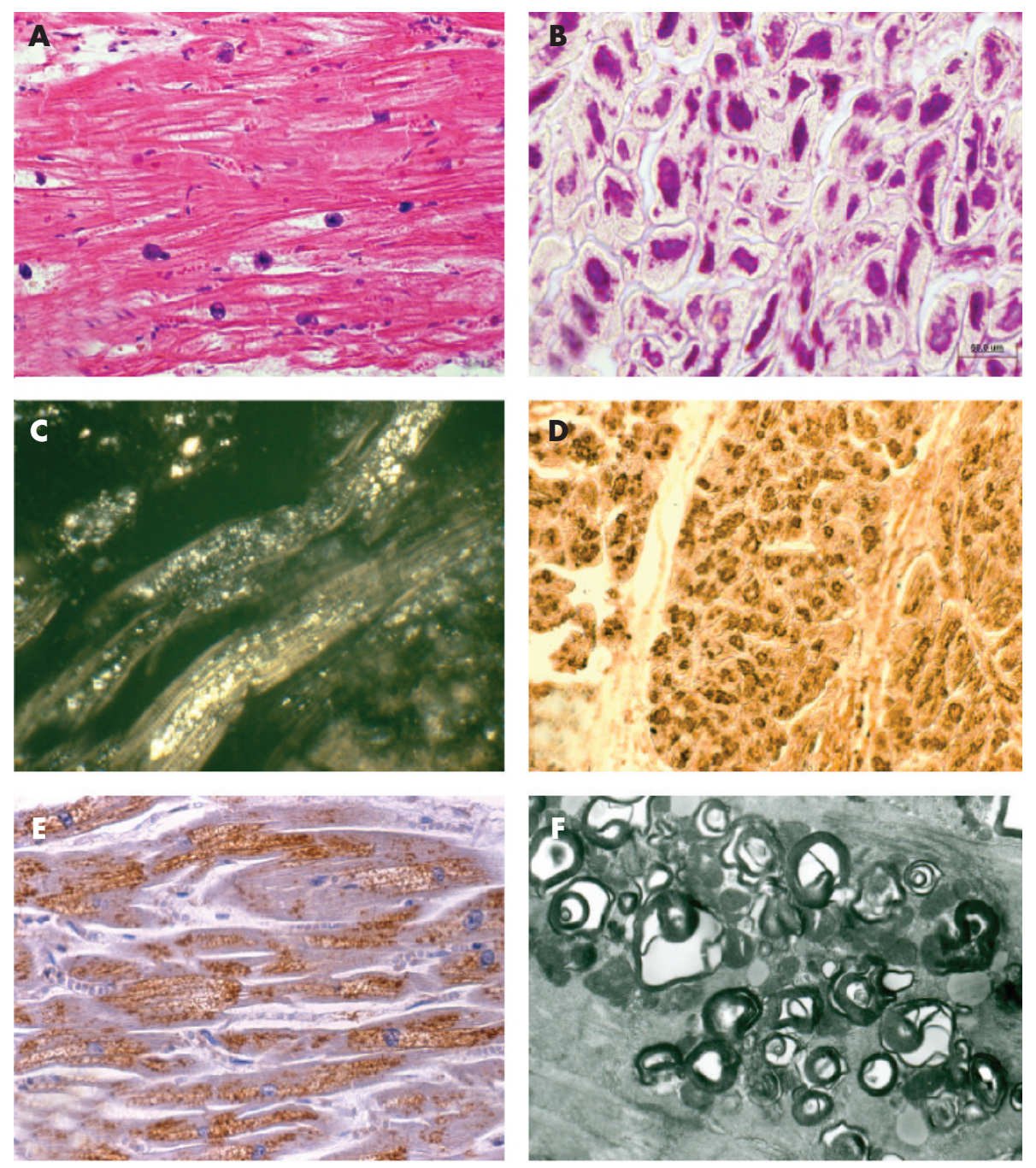

Figure 2 (A) Haematoxylin and eosin staining showing storage (perinuclear vacuoles) and hypertrophy of cardiomyocytes. (B) Glycolipid storage in cardiomyocytes demonstrated by periodic acid-Schiff (PAS) staining of a frozen section. (C) Prominent

birefringence of the stored glycolipid liquid crystals. (D) Immunohistochemical staining of the stored glycolipid using antibody against $\mathrm{Gb}_{3} \mathrm{Cer}$. (E) Prominent staining for luminal lysosomal marker cathepsin D in cardiocytes. (F) Electron microscopic ultrastructure of lysosomal glycolipid deposits in a cardiomyocyte. Images courtesy of Milan Elleder, Charles University, Prague, Czech Republic. 

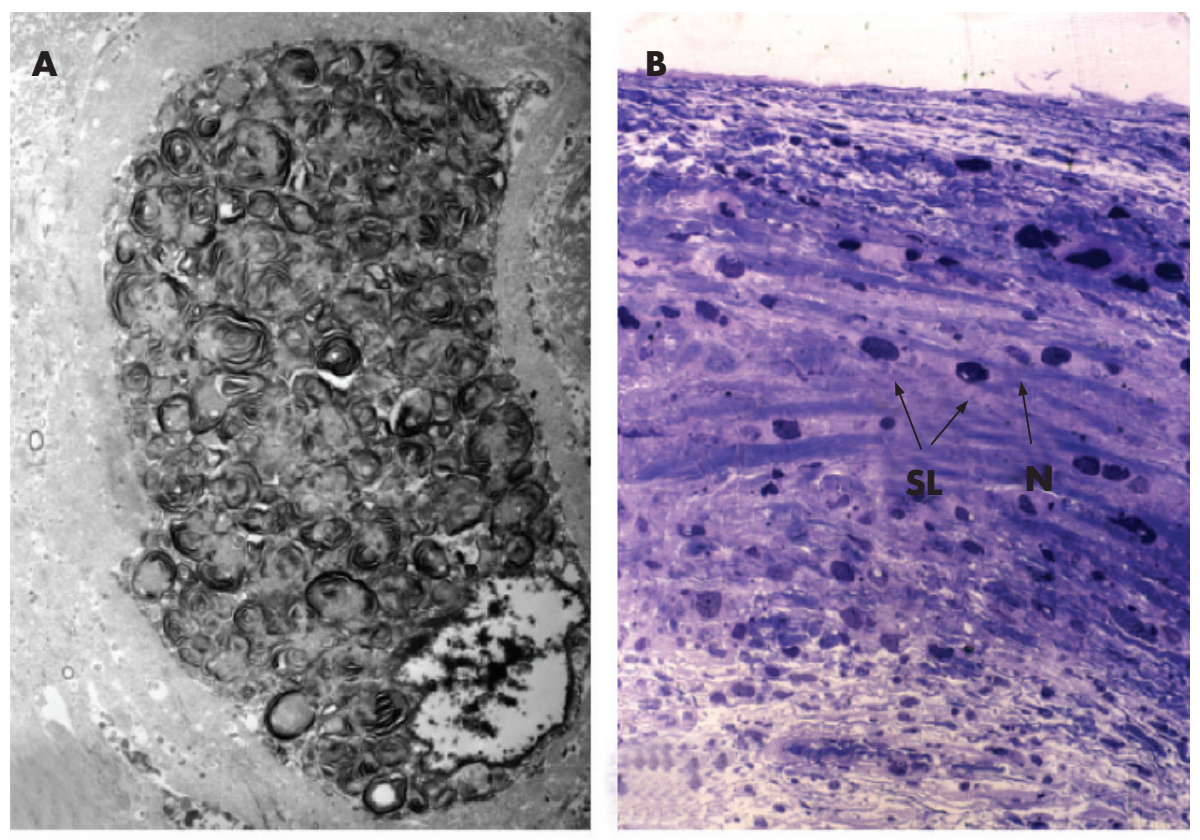

Figure 3 (A) Electron microscopic image of a valvular fibroblast loaded with stored glycolipid. (B) Semithin plastic embedded section of a mitral valve stained with alkaline toludene blue. Dark blue deposits demonstrate the stored lysosomal glycolipid (N, nuclei; SL, storage lysosome). (C)
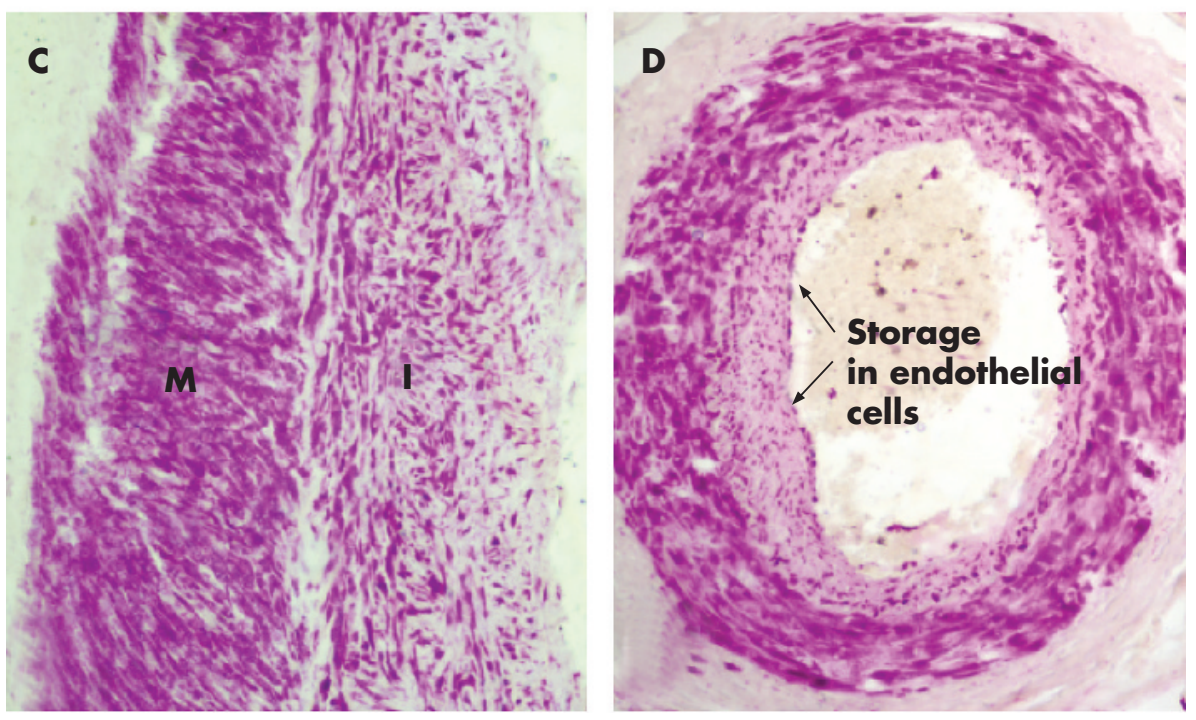

Sample of the left main coronary artery loaded with PAS + glycolipid in medial (M) and intimal (I) cells (longitudinal section). (D) Transverse section of a small subepicardial branch of a coronary artery loaded with PAS + glycolipid. Bulk of PAS positivity (panels C and D) was easily removed by preextraction using chloroform-methanol mixture. Images courtesy of Milan Elleder, Charles University, Prague, Czech Republic.

Table 3 Major clinical studies examining the prevalence of AFD among patients with unexplained left ventricular hypertrophy or hypertrophic cardiomyopathy

\begin{tabular}{|c|c|c|c|c|}
\hline Study & n, sex & Population & Age mean (SD) (range) & Prevalence $\mathrm{n}(\%)$ \\
\hline \multirow{3}{*}{$\begin{array}{l}\text { Nakao et } a^{2} \\
\text { Sachdev et } a^{\beta}\end{array}$} & 230 men & Consecutive, unexplained hypertrophy (IVS and/or PW $\geqslant 13 \mathrm{~mm}$ ) & $62(13)(55-72)$ & $7(3.0 \%)$ \\
\hline & 74 men & HCM diagnosed at $<40$ years of age (maximum wall thickness $\geqslant 13 \mathrm{~mm}$ ) & $26(9)(8-39)$ & $1(1.4 \%)$ \\
\hline & 79 men & $\mathrm{HCM}$ diagnosed at $\geqslant 40$ years of age (maximum wall thickness $\geqslant 13 \mathrm{~mm}$ ) & $53(8)(40-71)$ & $5(6.3 \%)$ \\
\hline \multirow[t]{3}{*}{ Arad et al ${ }^{11}$} & 75 (45 men) & Unexplained hypertrophy-HCM (wall thickness $\geqslant 13 \mathrm{~mm}$ ) & ND (12-75) & $0(0 \%)$ \\
\hline & 20 (NG) & $\mathrm{HCM}-$ massive LVH (wall thickness $\geqslant 30 \mathrm{~mm}$ ) & ND (9-58) & $0(0 \%)$ \\
\hline & 24 (NG) & HCM with pre-excitations (short PR, delta wave) & ND (8-42) & $0(0 \%)$ \\
\hline Ommen et al & 100 (44 men) & $\begin{array}{l}\text { Consecutive patients referred for septal myotomy-myectomy for LVOT } \\
\text { obstruction }\end{array}$ & 45 (17) at diagnosis (ND) & $0(0 \%)$ \\
\hline Chimenti et al ${ }^{4}$ & $\begin{array}{l}34 \text { women } \\
62 \text { men }\end{array}$ & $\begin{array}{l}\text { Consecutive, unexplained LVH (wall thickness } \geqslant 13 \mathrm{~mm} \text { ) } \\
\text { Unexplained LVH (wall thickness } \geqslant 13 \mathrm{~mm} \text { ) }\end{array}$ & $\begin{array}{l}50(14)(N D) \\
N D\end{array}$ & $\begin{array}{l}4(12 \%) \\
2(3.3 \%)\end{array}$ \\
\hline
\end{tabular}

HCM, hypertrophic cardiomyopathy; IVS, interventricular septum; LVH, left ventricular hypertrophy; LVOT, left ventricular oufflow tract; ND, no data; NG, not given; PW, posterior wall. 


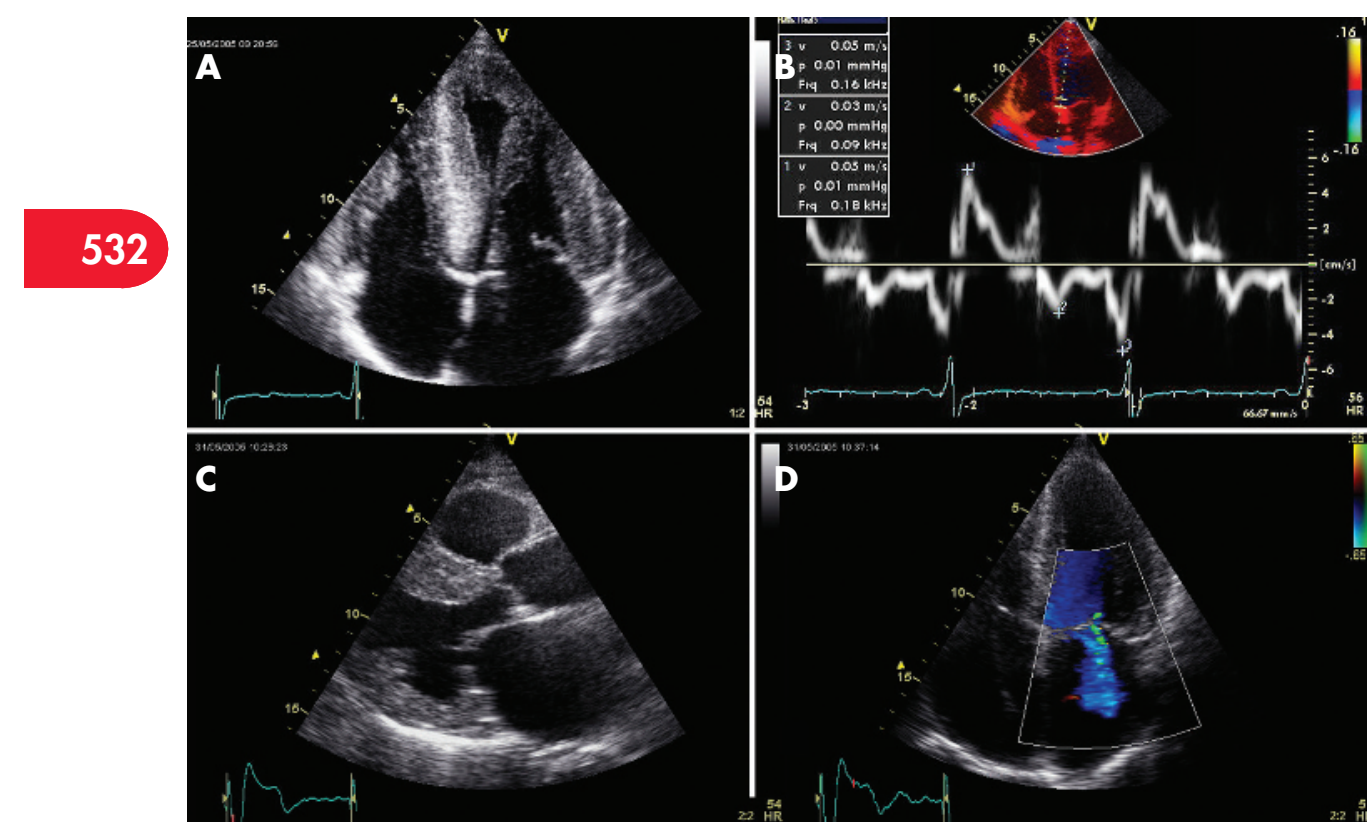

Figure 4 Echocardiographic images illustrating typical findings in patients with Anderson-Fabry disease. (A) Apical four-chamber view illustrating a severe left ventricular hypertrophy, right ventricular hypertrophy and mild bilateral atrial dilatation. (B) Tissue Doppler recording of the septal annular mitral velocities showing a significantly decreased early diastolic and systolic annular velocity. (C) Parasternal long axis view showing moderate left ventricular hypertrophy, mitral valve thickening and moderate left atrial dilatation. (D) Colour Doppler imaging in the same patient as on panel C, illustrating the presence of the mild mitral regurgitation. Bilateral atrial dilatation is evident and pacemaker electrode is present within the right ventricle.

\section{Cardiomyopathy}

Most patients (male and female) with AFD develop LVH (fig 4). ${ }^{16}$ Early disease is characterised by concentric remodelling, progressing later to concentric hypertrophy. Asymmetric septal hypertrophy, indistinguishable from that seen in sarcomeric cardiomyopathies, accounts for 5\% of cases. Dynamic left ventricular outflow obstruction is rare, but does occur. ${ }^{369}$ The right ventricle is often affected, but this does not appear to have major functional or clinical consequences.

Conventional measures of left ventricular systolic function are usually within the normal range. ${ }^{6}$ Nonetheless, studies using tissue Doppler imaging and strain-rate imaging have demonstrated reductions in systolic performance, occurring earlier in the longitudinal than in the radial dimension. ${ }^{10}$ Mild diastolic dysfunction is a common feature of AFD, but rarely causes restrictive pathophysiology except in the most advanced stages of the disease associated with pronounced fibrosis. ${ }^{6}{ }^{7}$

\section{Valve disease}

Valve disease in AFD is caused by infiltrative changes within valvular fibroblasts (figs 3 and 4). Although pulmonary valve involvement is reported, clinically significant changes are most frequently found on left heart valves, probably due to higher haemodynamic stress. In early reports, mitral valve prolapse was said to be common, but recent studies using twodimensional imaging suggests that it is relatively rare. ${ }^{6}$ Typically, valves are thickened and distorted, resulting in mild-to-moderate regurgitation; severe valve disease requiring surgical correction is infrequent. Aortic root dilatation is a feature in some patients and may contribute to aortic valve insufficiency. ${ }^{6}$

\section{Myocardial ischaemia}

Early studies suggested a high frequency of ischaemic events in patients with AFD. Contemporary data from registries suggest a low incidence of myocardial infarction, but show that angina and chest pain are frequent, particularly in patients with LVH.
Studies using positron emission tomography have shown that patients with AFD and angiographically normal coronary arteries have reduced coronary flow reserve suggesting that microvascular angina may be responsible for exertional symptoms in some patients. ${ }^{8}$ Cases of vasospastic angina are also reported.

\section{Electrophysiological abnormalities}

Most adult patients with AFD have an abnormal resting ECG. The most common abnormalities are voltage criteria for LVH and repolarisation changes. Many patients have a short PR interval probably caused by accelerated AV conduction in the majority. With disease progression, patients develop bundle branch block, AV conduction delay and progressive sinus node dysfunction. ${ }^{11}$

Patients with AFD often complain of palpitations. The most frequently encountered rhythm abnormalities are supraventricular tachycardias, atrial fibrillation and atrial flutter. ${ }^{11}$ Nonsustained ventricular tachycardia during 24 hour Holter recordings occur in up to $8.3 \%$ of patients and individual cases of fatal malignant arrhythmias resistant to ICD implantation are reported.

\section{Anderson-Fabry disease: key points}

- Anderson-Fabry disease is an X-linked lysosomal storage disorder caused by deficient activity of the enzyme $\alpha$-galactosidase A

- Anderson-Fabry disease accounts for at least 3\% of unexplained left ventricular hypertrophy in middle aged men

- Disease in the heart is characterised by progressive left ventricular hypertrophy, myocardial fibrosis, and systolic and diastolic dysfunction

- Arrhythmia and conduction disease are common in older patients and may cause stroke and sudden death 


\section{Treatment of Anderson-Fabry disease General measures}

Patients with chest pain should receive conventional antianginal treatment with the proviso that $\beta$-blockers may aggravate the tendency of some patients to symptomatic bradycardia and atrioventricular (AV) conduction block. Our own experience suggests that dihydropyridine calcium channel blockers are relatively effective and safe. Antiplatelet treatment should be offered to all symptomatic patients.

Conventional treatment with ACE inhibitors and diuretics should be used in patients with evidence for systolic impairment; ACE inhibitors (or angiotensin receptor blockers) should be considered in any patient with proteinuria or renal insufficiency. Patients with advanced congestive heart failure may be candidates for heart transplantation, as the intrinsic enzyme production within the graft should prevent reoccurrence of disease.

Many patients in advanced stages of the disease may require pacemaker implantation due to conduction abnormalities. In patients with supraventricular rhythm disturbances, anticoagulant treatment should be initiated. Subjects with documented malignant ventricular arrhythmias may benefit from implantable cardioverter-defibrillator (ICD) implantation.

There are few data on the management of outflow tract obstruction in AFD, but septal alcohol ablation has been used to good effect. Other treatment modalities (surgical myectomy, dual chamber pacing) may also be appropriate in symptomatic individuals.

\section{Enzyme replacement therapy}

Two recombinant enzyme preparations are approved for the treatment of AFD in Europe. Agalsidase-alfa is produced in human fibroblasts and is administered in a dose of $0.2 \mathrm{mg} / \mathrm{kg}$ of body weight every second week in intravenous (iv) infusions of 40 min. Most patients do not require any premedication. ${ }^{12}$ Agalsidase-beta is produced in a Chinese hamster ovarian cell line, administered also on a biweekly basis, but in a dose of $1 \mathrm{mg} / \mathrm{kg}$ in a 4-6 h iv infusion. Premedication with paracetamol may be required to prevent infusion reactions. ${ }^{13}$

Enzyme uptake is mediated by mannose-6-phosphate, mannose and asialoglycoprotein receptors. Thus, the rate of cellular uptake and tissue specificity depend on the glycosylation and phosphorylation characteristics of the enzyme protein, which differ between the two commercially available enzymes as post-translational protein modifications are species specific. Antibodies have been detected in male patients following the administration of both preparations; their effect on treatment efficacy and tolerability is not clear.

Several trials have shown that both preparations improve neurological and renal manifestations of the disease as well as quality of life..$^{12} \mathrm{~A}$ phase 3 trial using agalsidase-alfa has demonstrated an improvement in QRS complex duration. ${ }^{12}$ and $\mathrm{Gb}_{3}$ clearance from vascular endothelial cells. ${ }^{13}$ Case reports and observational clinical studies have reported improvements in left ventricular structure and function with both treatments. ${ }^{10}{ }^{14}$ Definitive proof of the long term beneficial effects of enzyme replacement therapy on the heart are awaited.

As discussed previously, mutations in the $\alpha$-galactosidase A gene exert their effect on the substrate protein in a number of different ways, including abnormal protein folding. In cultured cells, galactose and other reversible competitive inhibitors of $\alpha$-galactosidase A act as chemical "chaperones" that stabilise the enzyme sufficiently to allow its egress from the endoplasmic reticulum and transportation to the lysosome. Once in the lysosome, these inhibitors are competitively displaced from the active binding site by glycosphingolipid. In a single report of a middle aged male with cardiac variant disease, treatment with intravenous galactose (a competitive inhibitor of $\alpha$-galactosidase A) resulted in an improvement in symptoms and left ventricular function. ${ }^{15}$ The therapeutic effect of more easily administered chaperones is being investigated.

\section{CARDIAC DISEASE IN OTHER LYSOSOMAL STORAGE DISORDERS \\ Glycogen storage diseases}

While glycogen synthesis occurs only in the cellular cytoplasm, degradation occurs in cytoplasm and in the lysosomal compartment. These degradation pathways are not functionally connected and decreased activity of an enzyme in one or other pathway leads either to lysosomal or cytoplasmic storage. Lysosomal storage associated with cardiac involvement is found in GSD type IIa and IIb (Pompe and Danon disease, respectively).

\section{Pompe disease}

Pompe disease (synonyms: acid maltase deficiency, glycogen storage disease IIa) is an autosomal recessive disorder with infantile, juvenile and adult variants, each differing with respect to the age of onset, rate of disease progression and extent of tissue involvement. The infantile and childhood forms are characterised by myocardial glycogen deposition, massive cardiac hypertrophy and heart failure. The infantile form presents in the first few months of life with severe skeletal muscle hypotonia, progressive weakness, cardiomegaly, hepatomegaly and macroglossia. Left ventricular outflow obstruction is described in about $6 \%$ of patients. ${ }^{16}$ The ECG typically shows broad high-voltage QRS complexes and short PR interval with a pre-excitation pattern. The disease is usually fatal before 2 years of age due to cardiorespiratory failure. In the juvenile and adult onset variants it is usually limited to skeletal muscle, with a slowly progressive proximal myopathy and respiratory muscle weakness. ${ }^{16}$ Recent studies using recombinant enzyme replacement in the infantile and childhood forms have shown LVH regression and improved survival. ${ }^{17}$

\section{Danon disease}

Danon disease (synonyms: LAMP-2 deficiency, glycogen storage disorder IIb) is an X-linked deficiency of lysosome associated membrane protein type 2 (LAMP-2). The age and presentation of the disease is variable, with later onset and milder severity in heterozygous females. The typical phenotype of the disease includes skeletal myopathy, mental retardation and cardiomyopathy. Early death in affected males occurs usually between the second and third decade. Cardiac involvement is characterised by LVH, pre-excitation pattern and congestive heart failure. The pathological hallmark of the disease is the presence of intracytoplasmic vacuoles containing autophagic material and glycogen in cardiac and skeletal muscle cells. Disease clinically confined to the heart is described in some cases with later onset. ${ }^{18}$ 


\section{Mucopolysaccharidoses}

Mucopolysaccharidoses (MPS) are a large group of storage diseases caused by a defect of intralysosomal degradation of acid mucopolysaccharides (glycosaminglycans). Seven main forms and several subtypes can be distinguished. Cardiac involvement is detectable in more than two thirds of affected

children. The most common abnormalities occur in the valves with mitral and aortic valve thickening resulting in regurgitation and stenosis. The overall prevalence of valve abnormalities ranges from $27-90 \%$ depending on disease subtype (highest frequency in MPS I, II and VI, lowest in type IV and III). As in most lysosomal diseases, the involvement is progressive with age. ${ }^{19}$ Coronary involvement with intimal infiltration by storage cells is reported in more than $40 \%$ of patients, although rarely results in clinical or electrocardiographic evidence of myocardial ischaemia. The aorta may also be affected. ${ }^{19}$

Myocardial changes in MPS include interstitial infiltration and fibrosis. LVH, dilated cardiomyopathy and endomyocardial fibroelastosis are described. Cases of conduction system dysfunction are also reported and sudden cardiac deaths are relatively frequent. Enzyme replacement therapy is available for MPS type I, II and VI.

\section{Sphingolipidoses \\ Gaucher disease}

Gaucher disease is the most frequent sphingolipidosis. It is caused by a deficiency in $\beta$-glucocerebrosidase leading to lysosomal accumulation of glucocerebroside within macrophages. Lipid-laden macrophages (Gaucher cells) accumulate within the reticulo-endothelial system resulting in hepatosplenomegaly, bone marrow replacement, anaemia and thrombocytopenia and skeletal abnormalities. The disease is subclassified according to the onset and severity of the neurological involvement. Valvular and aortic calcification, heart failure and pericarditis are reported but the heart is not involved in most patients. More importantly, pulmonary hypertension occurs in up to $30 \%$ of untreated patients. Successful treatment appears to reduce the prevalence to $7.4 \%{ }^{20}$

\section{Niemann-Pick disease}

Niemann-Pick disease is caused by a deficiency in acid sphingomyelinase. It is subclassified into type A (infantile, neurodegenerative) and type B (later onset of hepatosplenomegaly, pulmonary involvement, survival into adulthood). Niemann-Pick types C and D represent distinct genetic and metabolic defects. Cardiac involvement is rare. ${ }^{20}$

\section{CONCLUSIONS}

Anderson-Fabry disease is an important differential diagnosis in middle aged and elderly patients with unexplained LVH. Emerging evidence suggests that enzyme replacement therapy substantially improves many of the features of the disease, including some aspects of cardiac involvement. The advent of new enzyme replacement therapies offer the prospect of a similar improvement in quality of life and prognosis for patients with other lysosomal storage disorders.

Additional references appear on the Heart website-http:// heart.bmj.com/supplemental

\section{INTERACTIVE MULTIPLE CHOICE QUESTIONS}

This education in Heart article has an accompanying series of six EBAC accredited multiple choice questions (MCQs).

To access the questions, click on BMJ Learning: Take this module on BMJ Learning from the content box at the top right and bottom left of the online article.

For more information please go to http://heart.bmj.com/misc/ education.dtl

Please note: The MCQs are hosted on BMJ Learning-the best available learning website for medical professionals from the BMJ Group.

If prompted, subscribers must sign into Heart with their journal's username and password. All users must also complete a one-time registration on BMJ Learning and subsequently log in (with a BMJ Learning username and password) on every visit.

\section{Authors' affiliations}

Aleš Linhart, $1^{\text {st }}$ School of Medicine, Charles University, Prague, Czech Republic

Perry M Elliott, The Heart Hospital, University College London, UK

In compliance with EBAC/EACCME guidelines, all authors participating in Education in Heart have disclosed potential conflicts of interest that might cause a bias in the article. Competing interest statement: $\mathrm{Dr}$ Elliott and $\mathrm{Dr}$ Linhart have received speaker and consultancy fees from TKT Inc (Shire Human Genetics Therapies) and Genzyme Inc. Dr Elliott is in receipt of an unrestricted educational grant from Shire Human Genetics Therapies. Neither author has any other financial conflict of interest to declare.

\section{REFERENCES}

1 Mehta A, Ricci R, Widmer U, et al. Fabry disease defined: baseline clinical manifestations of 366 patients in the Fabry Outcome Survey. Eur J Clin Invest 2004;34:236-42.

- Analysis of the largest ever published dataset of Anderson-Fabry disease patients. Comprehensive overview of disease key manifestations, prevalence and significance.

2 Nakao S, Takenaka T, Maeda M, et al. An atypical variant of Fabry's disease in men with left ventricular hypertrophy. N EnglJ Med 1995;333:288-93.

- First large study analysing the prevalence of Anderson-Fabry disease among patients with unexplained cardiac hypertrophy.

3 Sachdev B, Takenaka T, Teraguchi H, et al. Prevalence of Anderson-Fabry disease in male patients with late onset hypertrophic cardiomyopathy. Circulation 2002; 105: 1407-11

- Key study indicating larger prevalence of Anderson-Fabry disease among patients with late onset hypertrophic cardiomyopathy.

4 Chimenti C, Pieroni M, Morgante E, et al. Prevalence of Fabry disease in female patients with late-onset hypertrophic cardiomyopathy. Circulation 2004; 110:1047-53

5 Dobrovolny R, Dvorakova L, Ledvinova J, et al. Relationship between Xinactivation and clinical involvement in Fabry heterozygotes. Eleven novel mutations in the $\alpha$-galactosidase A gene in the Czech and Slovak population. J Mol Med 2005;3:647-54.

6 Linhart A, Palecek T, Bultas J, et al. New insights in cardiac structural changes in patients with Fabry's disease. Am Heart J 2000;139:1101-8.

- Echocardiographic data on left ventricular structure and function showing the high prevalence of concentric cardiac hypertrophy and remodelling.

7 Moon JC, Sachdev B, Elkington AG, et al. Gadolinium enhanced cardiovascular magnetic resonance in Anderson-Fabry disease. Evidence for a disease specific abnormality of the myocardial interstitium. Eur Heart J 2003;24:2151-5.

- First study demonstrating fibrotic changes within the basal posterolateral cardiac segments using the gadolinium enhanced magnetic resonance imaging.

8 Elliott PM, Kindler H, Shah JS, et al. Coronary microvascular dysfunction in male patients with Anderson-Fabry disease and the effect of treatment with $\alpha$ galactosidase A. Heart 2006;92:357-60.

- Study demonstrating the decrease of coronary flow reserve in patients with Anderson-Fabry disease.

9 Ommen SR, Nishimura RA, Edwards WD. Fabry disease: a mimic for obstructive hypertrophic cardiomyopathy? Heart 2003;89:929-30.

10 Weidemann F, Breunig F, Beer $M$, et al. Improvement of cardiac function during enzyme replacement therapy in patients with Fabry disease: a prospective strain rate imaging study. Circulation 2003;108:1299-301. 
11 Shah JS, Hughes DA, Sachdev B, et al. Prevalence and clinical significance of cardiac arrhythmia in Anderson-Fabry Disease. Am J Cardiol 2005:96:842-6.

- First report indicating high prevalence of supraventricular and ventricular arrhythmias in Anderson-Fabry disease and their relationship to left ventricular structure.

12 Schiffmann R, Kopp JB, Austin HA 3rd, et al. Enzyme replacement therapy in Fabry disease: a randomized controlled trial. JAMA 2001;285:2743-9.

- Key randomised study on enzyme replacement therapy with agalsidasealfa demonstrating clinical benefits on pain and renal function.

13 Eng CM, Guffon N, Wilcox WR, et al. International Collaborative Fabry Disease Study Group. Safety and efficacy of recombinant human alpha-galactosidase Areplacement therapy in Fabry's disease. N Engl J Med 2001;345:9-16.

- Key randomised study on enzyme replacement therapy with agalsidasebeta demonstrating significant clearance of $\mathrm{Gb} 3$ deposits, mainly from endothelial cells from organ biopsies.

14 Beck M, Ricci R, Widmer U, et al. Fabry disease: overall effects of agalsidase alfa treatment. Eur J Clin Invest 2004;34:838-44.

15 Frustaci A, Chimenti C, Ricci R, et al. Improvement in cardiac function in the cardiac variant of Fabry's disease with galactose-infusion therapy. N Engl J Med $2001 ; 345: 25-32$
16 van den Hout HPM, Hop W, van Diggelen OP, et al. The natural course of infantile Pompe's disease: 20 original cases compared with 133 cases from the literature. Pediatrics 2003;1 12:332-40.

17 Klinge L, Straub V, Neudorf U, et al. Safety and efficacy of recombinant acid alpha-glucosidase (rhGAA) in patients with classical infantile Pompe disease: results of a phase II clinical trial. Neuromuscul Disord 2005;15:24-31.

18 Arad M, Maron BJ, Gorham JM, et al. Glycogen storage diseases presenting as hypertrophic cardiomyopathy. N Engl J Med 2005;352:362-72.

19 Mohan UR, Hay AA, Cleary MA, et al. Cardiovascular changes in children with mucopolysaccharide disorders. Acta Paediatr 2002:91:799-804.

20 Guertl B, Noehammer C, Hoefler G. Metabolic cardiomyopathies. Int J Exp Pathol 2000;81:349-72.

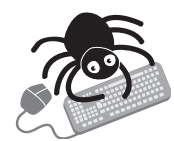

Additional references appear on the Heart websitehttp://heart.bmi.com/supplemental

\section{MULTIPLE CHOICE QUESTIONS}

\section{Education in Heart Interactive (heart.bmi.com/misc/education.dtl)}

Education in Heart (EiH) articles each have an accompanying series of six multiple choice questions. These are hosted on BMJ Learning - the best available learning website for medical professionals from the BMJ Group. Each article is submitted to EBAC (European Board for Accreditation in Cardiology; ebac-cme.org) for 1 hour of external CPD credit.

Free access for subscribers: For full details of the resources available to subscribers please see: heart.bmj.com/misc/education.dtl\#access

How to access the questions: Click on BMJ Learning: Take this module on BMJ Learning from the online article content box, table of contents or EiH collection

(heart.bmj.com/cgi/collection/heart_education).

If prompted, subscribers must sign into Heart with their journal's username and password.

Please note: All users must also complete a one-time registration on BMJ Learning. Users will then subsequently log in (with a BMJ Learning username and password) on every visit in order to log activity and provide appropriate access.

Activating your subscription to Heart: If you have not yet activated your online subscription to Heart, please visit journals.bmj.com/cgi/activate/basic and enter your six digit (all numeric) customer number (found above your address label with your print copy). If you have any queries, please contact subscriptions@bmigroup.com

Case based learning: You may also be interested in the cardiology interactive case histories published in association with Heart, for more information please see:

heart.bmi.com/misc/education.dtl\#ichs 